\title{
BOUNDED ELEMENTS OF C*-INDUCTIVE LOCALLY CONVEX SPACES
}

\author{
GIORGIA BELLOMONTE, SALVATORE DI BELLA, AND CAMILLO TRAPANI
}

\begin{abstract}
The notion of bounded element of $\mathrm{C}^{*}$-inductive locally convex spaces (or $\mathrm{C}^{*}$-inductive partial *-algebras) is introduced and discussed in two ways: the first one takes into account the inductive structure provided by certain families of $\mathrm{C}^{*}$-algebras; the second one is linked to natural order of these spaces. A particular attention is devoted to the relevant instance provided by the space of continuous linear maps acting in a rigged Hilbert space.
\end{abstract}

\section{INTRODUCTION}

Some locally convex spaces exhibit an interesting feature: they contain a large number of $\mathrm{C}^{*}$-algebras that often contribute to their topological structure, in the sense that these spaces can be thought as generalized inductive limits of $\mathrm{C}^{*}$-algebras. These objects were called $C^{*}$-inductive locally convex spaces in [8] and their structure was examined in detail, also taking in mind that they arise naturally when one considers the operators acting in the joint topological limit of an inductive family of Hilbert spaces as described in [7]. Indeed, a typical instance of this structure is obtained by considering the space $\mathfrak{L}_{\mathrm{B}}\left(\mathcal{D}, \mathcal{D}^{\times}\right)$of operators acting in the rigged Hilbert space canonically associated to an $\mathrm{O}^{*}$-algebra of unbounded operators acting on a dense domain $\mathcal{D}$ of Hilbert space $\mathcal{H}$. In [8] a series of features of this structure were studied giving a particular attention to the order structure, positive linear functionals and representation theory. The space $\mathfrak{L}_{\mathrm{B}}\left(\mathcal{D}, \mathcal{D}^{\times}\right)$contains a subspace isomorphic to the ${ }^{*}$-algebra $\mathfrak{B}(\mathcal{H})$ of bounded operators in $\mathcal{H}$ whose elements can be in natural way considered as the bounded elements of $\mathfrak{L}_{\mathrm{B}}\left(\mathcal{D}, \mathcal{D}^{\times}\right)$. The notion of bounded element of a locally convex *-algebra $\mathfrak{A}$ was first introduced by Allan [1] with the aim of developing a spectral theory for topological *-algebras: an element $x$ of the topological *-algebra $\mathfrak{A}[\tau]$ is

2010 Mathematics Subject Classification. 47L60, 47L40.

Key words and phrases. bounded elements, inductive limit of $\mathrm{C}^{*}$-algebras, partial *algebras. 
Allan bounded if there exists $\lambda \neq 0$ such that the set $\left\{\left(\lambda^{-1} x\right)^{n} ; n=1,2, \ldots\right\}$ is a bounded subset of $\mathfrak{A}[\tau]$. This definition was suggested by the successful spectral analysis for closed operators in Hilbert space $\mathcal{H}$ : a complex number $\lambda$ is in the resolvent set $\rho(T)$ of a closed operator $T$ if $T-\lambda I$ has an inverse in the ${ }^{*}$-algebra $\mathfrak{B}(\mathcal{H})$ of bounded operators.

There are, however, several other possibilities for defining bounded elements. For instance, one may say that $x$ is bounded if $\pi(x)$ is a bounded operator, for every (continuous, in a certain sense) *-representation $\pi$ defined on a dense domain $\mathcal{D}_{\pi}$ of some Hilbert space $\mathcal{H}_{\pi}$. This could be a reasonable definition in itself, provided that $\mathfrak{A}$ possesses sufficiently many *-representations in Hilbert space.

Moreover some attempts to extend this notion to the larger set-up of locally convex quasi *-algebras [18, 20, 21, 11] or locally convex partial *algebras [4, 5, 6] have been done. But in these cases, Allan's notion cannot be adopted, since powers of a given element $x$ need not be defined.

In the case of *-algebras, bounded elements in purely algebraic terms have been considered by Vidav [23] and Schmüdgen [17] with respect to some (positive) wedge.

The aim of this paper is to extend the notion of bounded element to the case of $\mathrm{C}^{*}$-inductive locally convex spaces $\mathfrak{A}$ with defining family of $\mathrm{C}^{*}$ algebras $\left\{\mathfrak{B}_{\alpha} ; \alpha \in \mathbb{F}\right\}$ ( $\mathbb{F}$ is an index set directed upward). There are also in this case several possibilities: the first one consists in taking elements that have representatives in every $\mathrm{C}^{*}$-algebra $\mathfrak{B}_{\alpha}$ of the family whose norms are uniformly bounded; the second one consists into taking into account the order structure of $\mathfrak{A}$, in the same spirit of the quoted papers of Vidav and Schmüdgen.

The paper is organized as follows. After some preliminaries (Section 2), we study, in Section 3, how bounded elements of $\mathfrak{L}_{\mathrm{B}}\left(\mathcal{D}, \mathcal{D}^{\times}\right)$can be derived from its $\mathrm{C}^{*}$-inductive structure and from its order structure. We show that these two notions are equivalent and that an element $X$ is bounded if and only if $X$ maps $\mathcal{D}$ into $\mathcal{H}$ and $\bar{X} \in \mathfrak{B}(\mathcal{H})$. Finally, in Section 4 , we consider the same problem for abstract $\mathrm{C}^{*}$-inductive locally convex spaces and give conditions for some of the characterizations proved for $\mathfrak{L}_{\mathrm{B}}\left(\mathcal{D}, \mathcal{D}^{\times}\right)$maintain their validity. Some of these results are then specialized to the case where $\mathfrak{A}$ is a $\mathrm{C}^{*}$-inductive locally convex partial *-algebra. 


\section{Notations AND PRELIMINARIES}

For general aspects of the theory of partial *-algebras and of their representations, we refer to the monograph [2]. For the convenience of the reader, however, we repeat here the essential definitions.

A partial *-algebra $\mathfrak{A}$ is a complex vector space with conjugate linear involution * and a distributive partial multiplication ', defined on a subset $\Gamma \subset \mathfrak{A} \times \mathfrak{A}$, satisfying the property that $(x, y) \in \Gamma$ if, and only if, $\left(y^{*}, x^{*}\right) \in \Gamma$ and $(x \cdot y)^{*}=y^{*} \cdot x^{*}$. From now on, we will write simply $x y$ instead of $x \cdot y$ whenever $(x, y) \in \Gamma$. For every $y \in \mathfrak{A}$, the set of left (resp. right) multipliers of $y$ is denoted by $L(y)$ (resp. $R(y)$ ), i.e., $L(y)=\{x \in \mathfrak{A}:(x, y) \in \Gamma\}$, (resp. $R(y)=\{x \in \mathfrak{A}:(y, x) \in \Gamma\})$. We denote by $L \mathfrak{A}($ resp. $R \mathfrak{A})$ the space of universal left (resp. right) multipliers of $\mathfrak{A}$. In general, a partial *-algebra is not associative.

The unit of partial *-algebra $\mathfrak{A}$, if any, is an element $e \in \mathfrak{A}$ such that $e=e^{*}, e \in R \mathfrak{A} \cap L \mathfrak{A}$ and $x e=e x=x$, for every $x \in \mathfrak{A}$.

Let $\mathcal{H}$ be a complex Hilbert space and $\mathcal{D}$ a dense subspace of $\mathcal{H}$. We denote by $\mathrm{E}^{\dagger}(\mathcal{D}, \mathcal{H})$ the set of all (closable) linear operators $X$ such that $D(X)=\mathcal{D}, D\left(X^{*}\right) \supseteq \mathcal{D}$. The map $X \rightarrow X^{\dagger}=X_{\bullet \mathcal{D}}^{*}$ defines an involution on $\mathrm{E}^{\dagger}(\mathcal{D}, \mathcal{H})$, which can be made into a partial *-algebra with respect to the weak multiplication [2]; however, this fact will not be used in this paper.

Let $\mathcal{L}^{\dagger}(\mathcal{D})$ be the subspace of $\mathcal{L}^{\dagger}(\mathcal{D}, \mathcal{H})$ consisting of all its elements which leave, together with their adjoints, the domain $\mathcal{D}$ invariant. Then $\mathcal{L}^{\dagger}(\mathcal{D})$ is a *-algebra with respect to the usual operations. A *-subalgebra $\mathfrak{M}$ of $\mathcal{L}^{\dagger}(\mathcal{D})$ is called an $\mathrm{O}^{*}$-algebra.

Let $\mathfrak{M}$ be an $\mathrm{O}^{*}$-algebra. The graph topology $t_{\mathfrak{M}}$ on $\mathcal{D}$ is the locally convex topology defined by the family $\left\{\|\cdot\|_{A}\right\}_{A \in \mathfrak{M}}$, where

$$
\|\xi\|_{A}=\sqrt{\|\xi\|^{2}+\|A \xi\|^{2}}=\left\|\left(I+A^{*} \bar{A}\right)^{1 / 2} \xi\right\|, \quad \xi \in \mathcal{D} .
$$

For $A=0$, the null operator of $\mathcal{L}^{\dagger}(\mathcal{D}),\|\cdot\|_{0}$ is exactly the norm of $\mathcal{H}$, thus we will omit the 0 in the notation of the norm.

The topology $t_{\mathfrak{M}}$ is finer than the norm topology, unless $\mathfrak{M}$ does consist of bounded operators only.

If $\mathfrak{M}$ is an $\mathrm{O}^{*}$-algebra, we write $A \preceq B$ if $\|A \xi\| \leq\|B \xi\|$, for every $\xi \in \mathcal{D}$. Then, $\mathfrak{M}$ is directed upward with respect to this order relation.

If $A \in \mathfrak{M}$, we denote by $\mathcal{H}_{A}$ the Hilbert space obtained by endowing $D(\bar{A})$ with the graph norm $\|\cdot\|_{A}$. 
If $A, B \in \mathfrak{M}$ and $A \preceq B$, then $U_{B A}=\left(I+B^{*} \bar{B}\right)^{-1 / 2}\left(I+A^{*} \bar{A}\right)^{1 / 2}$ is a contractive map of $\mathcal{H}_{A}$ into $\mathcal{H}_{B}$; i.e., $\left\|U_{B A} \xi\right\|_{B} \leq\|\xi\|_{A}$, for every $\xi \in \mathcal{H}_{A}$.

If the locally convex space $\mathcal{D}\left[t_{\mathfrak{M}}\right]$ is complete, then $\mathfrak{M}$ is said to be closed.

If $\mathfrak{M}=\mathcal{L}^{\dagger}(\mathcal{D})$ then the corresponding graph topology is denoted by $t_{\dagger}$ instead of $t_{\mathcal{L}^{\dagger}(\mathcal{D})}$.

As is known, a locally convex topology $t$ on $\mathcal{D}$ finer than the topology induced by the Hilbert norm defines, in standard fashion, a rigged Hilbert space (RHS)

$$
\mathcal{D}[t] \hookrightarrow \mathcal{H} \hookrightarrow \mathcal{D}^{\times}\left[t^{\times}\right],
$$

where $\mathcal{D}^{\times}$is the vector space of all continuous conjugate linear functionals on $\mathcal{D}[t]$, i.e., the conjugate dual of $\mathcal{D}[t]$, endowed with the strong dual topology $t^{\times}=\beta\left(\mathcal{D}^{\times}, \mathcal{D}\right)$ and $\hookrightarrow$ denotes a continuous embedding with dense range. The Hilbert space $\mathcal{H}$ is identified (by considering the form which puts $\mathcal{D}$ and $\mathcal{D}^{\times}$as an extension of the inner product of $\mathcal{D}$ ) with a dense subspace of $\mathcal{D}^{\times}\left[t^{\times}\right]$.

Let $\mathfrak{L}\left(\mathcal{D}, \mathcal{D}^{\times}\right)$denote the vector space of all continuous linear maps from $\mathcal{D}[t]$ into $\mathcal{D}^{\times}\left[t^{\times}\right]$. In $\mathfrak{L}\left(\mathcal{D}, \mathcal{D}^{\times}\right)$an involution $X \mapsto X^{\dagger}$ can be introduced by the equality

$$
\langle X \xi \mid \eta\rangle=\overline{\left\langle X^{\dagger} \eta \mid \xi\right\rangle}, \quad \forall \xi, \eta \in \mathcal{D} .
$$

Hence $\mathfrak{L}\left(\mathcal{D}, \mathcal{D}^{\times}\right)$is a ${ }^{*}$-invariant vector space.

To every $X \in \mathfrak{L}\left(\mathcal{D}, \mathcal{D}^{\times}\right)$there corresponds a separately continuous sesquilinear form $\theta_{X}$ on $\mathcal{D} \times \mathcal{D}$ defined by

$$
\theta_{X}(\xi, \eta)=\langle X \xi \mid \eta\rangle, \quad \xi, \eta \in \mathcal{D} .
$$

The vector space of all jointly continuous sesquilinear forms on $\mathcal{D} \times \mathcal{D}$ will be denoted with $\mathrm{B}(\mathcal{D}, \mathcal{D})$. We denote by $\mathfrak{L}_{\mathrm{B}}\left(\mathcal{D}, \mathcal{D}^{\times}\right)$the subspace of all $X \in$ $\mathfrak{L}\left(\mathcal{D}, \mathcal{D}^{\times}\right)$such that $\theta_{X} \in \mathbf{B}(\mathcal{D}, \mathcal{D})$ and by $\mathfrak{L}^{\dagger}(\mathcal{D})$ the ${ }^{*}$-algebra consisting of all operators of $\mathcal{L}^{\dagger}(\mathcal{D})$, which together with their adjoints are continuous from $\mathcal{D}[t]$ into $\mathcal{D}[t]$. If $t=t_{\dagger}$, then $\mathfrak{L}^{\dagger}(\mathcal{D})=\mathcal{L}^{\dagger}(\mathcal{D})$. We will refer to the rigged Hilbert space defined by endowing $\mathcal{D}$ with the topology $t_{\dagger}$ as to the canonical rigged Hilbert space defined by $\mathcal{L}^{\dagger}(\mathcal{D})$ on $\mathcal{D}$. In this case $\left(\mathfrak{L}_{\mathrm{B}}\left(\mathcal{D}, \mathcal{D}^{\times}\right), \mathcal{L}^{\dagger}(\mathcal{D})\right)$ is a quasi *-algebra [2].

The spaces $\mathfrak{L}\left(\mathcal{D}, \mathcal{D}^{\times}\right)$and $\mathfrak{L}_{\mathrm{B}}\left(\mathcal{D}, \mathcal{D}^{\times}\right)$have been studied at length by several authors (see, e.g. [12, 13, 14, 22]) and several pathologies concerning their multiplicative structure have been considered (see also [2, 3] and references therein). Recently some spectral properties of operators of these classes have also been studied [10]. 


\section{Bounded Elements of $\mathfrak{L}_{\mathrm{B}}\left(\mathcal{D}, \mathcal{D}^{\times}\right)$}

The inductive structure of $\mathfrak{L}_{\mathrm{B}}\left(\mathcal{D}, \mathcal{D}^{\times}\right)$, with $\mathcal{D}$ endowed with the graph topology $t_{\dagger}$, has been discussed in [8, Section 5$]$. To keep the paper reasonably self-contained, we sum the main features up.

By the definition itself, $X \in \mathfrak{L}_{\mathrm{B}}\left(\mathcal{D}, \mathcal{D}^{\times}\right)$if, and only if, there exists $\gamma_{X}>0$ and $A \in \mathcal{L}^{\dagger}(\mathcal{D})$ such that

$$
\left|\theta_{X}(\xi, \eta)\right|=|\langle X \xi \mid \eta\rangle| \leq \gamma_{X}\|\xi\|_{A}\|\eta\|_{A}, \quad \forall \xi, \eta \in \mathcal{D} .
$$

Conversely, if $\theta \in \mathrm{B}(\mathcal{D}, \mathcal{D})$, there exists a unique $X \in \mathfrak{L}_{\mathrm{B}}\left(\mathcal{D}, \mathcal{D}^{\times}\right)$such that $\theta=\theta_{X}$.

Thus, the map

$$
\mathbb{I}: X \in \mathfrak{L}_{\mathrm{B}}\left(\mathcal{D}, \mathcal{D}^{\times}\right) \mapsto \theta_{X} \in \mathrm{B}(\mathcal{D}, \mathcal{D})
$$

is an isomorphism of vector spaces and $\mathbb{I}\left(\theta^{*}\right)=X^{\dagger}$, where $\theta^{*}(\xi, \eta)=\overline{\theta(\eta, \xi)}$, for every $\xi, \eta \in \mathcal{D}$.

We denote by $\mathrm{B}^{A}(\mathcal{D}, \mathcal{D})$ the subspace of $\mathrm{B}(\mathcal{D}, \mathcal{D})$ consisting of all $\theta \in$ $\mathrm{B}(\mathcal{D}, \mathcal{D})$ such that (1) holds for fixed $A \in \mathcal{L}^{\dagger}(\mathcal{D})$.

If $\theta \in \mathrm{B}^{A}(\mathcal{D}, \mathcal{D})$, it extends to a bounded sesquilinear form on $\mathcal{H}_{A} \times \mathcal{H}_{A}$ (we use the same symbol for this extension). Hence, there exists a unique operator $X_{A}^{\theta} \in \mathfrak{B}\left(\mathcal{H}_{A}\right)$ such that

$$
\theta(\xi, \eta)=\left\langle X_{A}^{\theta} \xi \mid \eta\right\rangle_{A}, \quad \forall \xi, \eta \in \mathcal{H}_{A}
$$

On the other hand, if $X_{A} \in \mathfrak{B}\left(\mathcal{H}_{A}\right)$, then the sesquilinear form $\theta_{X_{A}}$ defined by

$$
\theta_{X_{A}}(\xi, \eta)=\left\langle X_{A} \xi \mid \eta\right\rangle_{A}, \quad \xi, \eta \in \mathcal{D}
$$

is an element of $\mathrm{B}^{A}(\mathcal{D}, \mathcal{D})$ and the map

$$
\Phi_{A}: X_{A} \in \mathfrak{B}\left(\mathcal{H}_{A}\right) \rightarrow \theta_{X_{A}} \in \mathrm{B}^{A}(\mathcal{D}, \mathcal{D})
$$

is a ${ }^{*}$-isomorphism of vector spaces with involution.

If $B \succeq A$, then, for $\xi, \eta \in \mathcal{D}$,

$$
\left|\theta_{X_{A}}(\xi, \eta)\right|=\left|\left\langle X_{A} \xi \mid \eta\right\rangle_{A}\right| \leq\left\|X_{A}\right\|_{A, A}\|\xi\|_{A}\|\eta\|_{A} \leq\left\|X_{A}\right\|_{A, A}\|\xi\|_{B}\|\eta\|_{B},
$$

where $\|\cdot\|_{A, A}$ denotes the operator norm in $\mathfrak{B}\left(\mathcal{H}_{A}\right)$. Hence, there exists a unique $X_{B} \in \mathfrak{B}\left(\mathcal{H}_{B}\right)$ such that

$$
\left\langle X_{A} \xi \mid \eta\right\rangle_{A}=\left\langle X_{B} \xi \mid \eta\right\rangle_{B}, \quad \forall \xi, \eta \in \mathcal{D} .
$$

So it is natural to define

$$
J_{B A}\left(X_{A}\right)=X_{B}, \quad \forall X_{A} \in \mathfrak{B}\left(\mathcal{H}_{A}\right) .
$$


It is easily seen that $J_{B A}=\Phi_{B}^{-1} \Phi_{A}$.

The space $\mathfrak{L}_{\mathrm{B}}^{A}\left(\mathcal{D}, \mathcal{D}^{\times}\right):=\mathbb{I}^{-1} \mathrm{~B}^{A}(\mathcal{D}, \mathcal{D})$ is a Banach space, with norm

$$
\|X\|^{A}:=\sup _{\|\xi\|_{A},\|\eta\|_{A} \leq 1}\left|\theta_{X}(\xi, \eta)\right|
$$

and $\mathfrak{L}_{\mathrm{B}}\left(\mathcal{D}, \mathcal{D}^{\times}\right)$can be endowed with the inductive topology $\tau_{\text {ind }}$ defined by the family of subspaces $\left\{\mathfrak{L}_{\mathrm{B}}^{A}\left(\mathcal{D}, \mathcal{D}^{\times}\right) ; A \in \mathcal{L}^{\dagger}(\mathcal{D})\right\}$ as in [16, Section 1.2. III].

In conclusion,

$$
X_{A} \in \mathfrak{B}\left(\mathcal{H}_{A}\right) \leftrightarrow \theta_{X_{A}} \in \mathrm{B}^{A}(\mathcal{D}, \mathcal{D}) \leftrightarrow X \in \mathfrak{L}_{\mathrm{B}}^{A}\left(\mathcal{D}, \mathcal{D}^{\times}\right)
$$

are isometric *-isomorphisms of Banach spaces.

Hence, to every $X \in \mathfrak{L}_{\mathrm{B}}\left(\mathcal{D}, \mathcal{D}^{\times}\right)$one can associate the net $\left\{X_{B} ; B \in\right.$ $\left.\mathcal{L}^{\dagger}(\mathcal{D}) ; B \succeq A\right\}$ of its representatives in each of the spaces $\mathcal{H}_{B}$.

Definition 3.1. We say that $X \in \mathfrak{L}_{\mathrm{B}}\left(\mathcal{D}, \mathcal{D}^{\times}\right)$is a bounded element of $\mathfrak{L}_{\mathrm{B}}\left(\mathcal{D}, \mathcal{D}^{\times}\right)$if $X$ has a representative $X_{A}$ in every $\mathfrak{B}\left(\mathcal{H}_{A}\right)$ and

$$
\|X\|_{b}:=\sup _{A \in \mathcal{L}^{\dagger}(\mathcal{D})}\left\|X_{A}\right\|_{A, A}<+\infty .
$$

The space $\mathfrak{L}_{\mathrm{B}}\left(\mathcal{D}, \mathcal{D}^{\times}\right)_{b}$ of all bounded elements of $\mathfrak{L}_{\mathrm{B}}\left(\mathcal{D}, \mathcal{D}^{\times}\right)$is a Banach space with norm $\|\cdot\|_{b}$.

Proposition 3.2. $\mathfrak{L}_{B}\left(\mathcal{D}, \mathcal{D}^{\times}\right)_{b}$ is ${ }^{*}$-isomorphic (as Banach space) to a $C^{*}$ algebra of operators.

Proof. Let $\mathcal{H}_{\oplus}$ denote the Hilbert space direct sum of the $\mathcal{H}_{A}, A \in \mathcal{L}^{\dagger}(\mathcal{D})$; i.e.,

$$
\begin{aligned}
\mathcal{H}_{\oplus} & :=\bigoplus_{A \in \mathcal{L}^{\dagger}(\mathcal{D})} \mathcal{H}_{A} \\
& =\left\{\xi_{\oplus}=\left(\xi_{A}\right) ; \xi_{A} \in \mathcal{H}_{A}, \forall A \in \mathcal{L}^{\dagger}(\mathcal{D}) \text { and } \sum_{A}\left\|\xi_{A}\right\|_{A}^{2}<+\infty\right\} .
\end{aligned}
$$

If $\left\{X_{A}\right\}_{A \in \mathcal{L}^{\dagger}(\mathcal{D})}$ is a net of operators $X_{A} \in \mathfrak{B}\left(\mathcal{H}_{A}\right), A \in \mathcal{L}^{\dagger}(\mathcal{D})$, we define $X_{\oplus} \xi_{\oplus}=\left\{X_{A} \xi_{A}\right\}$ provided that $\sum_{A}\left\|X_{A} \xi_{A}\right\|^{2}<+\infty, \xi_{A} \in \mathcal{H}_{A}$.

The operator $X_{\oplus}=\left\{X_{A}\right\}$ is bounded if and only if $\sup _{A}\left\|X_{A}\right\|_{A, A}<+\infty$. The space constructed in this way is $\prod_{A} \mathfrak{B}\left(\mathcal{H}_{A}\right)=\mathfrak{B}\left(\mathcal{H}_{\oplus}\right)$. To every $X \in$ $\mathfrak{L}_{\mathrm{B}}\left(\mathcal{D}, \mathcal{D}^{\times}\right)_{b}$ we can associate the net $\left\{X_{A}\right\}$ which we have defined above. Clearly, $\left\{X_{A}\right\} \in \mathfrak{B}\left(\mathcal{H}_{\oplus}\right)$. It is easily seen that the map

$$
\tau: X \in \mathfrak{L}_{\mathrm{B}}\left(\mathcal{D}, \mathcal{D}^{\times}\right)_{b} \mapsto\left\{X_{A}\right\} \in \mathfrak{B}\left(\mathcal{H}_{\oplus}\right)
$$

is isometric. Thus, the statement is proved. 
Remark 3.3. An element $X \in \mathfrak{L}_{\mathrm{B}}\left(\mathcal{D}, \mathcal{D}^{\times}\right)$having a representative $X_{A}$ for every $A \in \mathcal{L}^{\dagger}(\mathcal{D})$ need not be bounded in the sense of Definition 3.1. The spaces $\left\{\mathcal{H}_{A} ; A \in \mathcal{L}^{\dagger}(\mathcal{D})\right\}$, together with their conjugate duals make $D^{\times}$into an indexed PIP-space [3, Ch.2]. In that language, operators having representatives in every $\mathcal{H}_{A}$ are called totally regular operators. For more details on their behavior see [3, Sect. 3.3.3] where also a $\mathrm{C}^{*}$-agebra corresponding to our bounded elements has been studied.

Our next goal is to characterize bounded elements of $\mathfrak{L}_{\mathrm{B}}\left(\mathcal{D}, \mathcal{D}^{\times}\right)$in several different ways. For doing this, we need to consider the natural order structure of $\mathfrak{L}_{\mathrm{B}}\left(\mathcal{D}, \mathcal{D}^{\times}\right)$.

We say that $X \in \mathfrak{L}_{\mathrm{B}}\left(\mathcal{D}, \mathcal{D}^{\times}\right)$is positive, and write $X \geq 0$, if $\langle X \xi \mid \xi\rangle \geq 0$, for every $\xi \in \mathcal{D}$.

It is easy to see that, if $X$ is positive, then it is symmetric; i.e., $X=X^{\dagger}$.

Proposition 3.4. The following conditions are equivalent.

(i) $X \geq 0$.

(ii) There exists $A \in \mathcal{L}^{\dagger}(\mathcal{D})$ such that $X_{B} \geq 0, \quad \forall B \succeq A$.

Proof. (i) $\Rightarrow$ (ii): Since $X \in \mathfrak{L}_{\mathrm{B}}\left(\mathcal{D}, \mathcal{D}^{\times}\right)$, there exists $A \in \mathcal{L}^{\dagger}(\mathcal{D})$ and $\gamma>0$ such that

$$
|\langle X \xi \mid \eta\rangle| \leq \gamma\|\xi\|_{B}\|\eta\|_{B}, \quad B \succeq A .
$$

If $X \geq 0$, then, for every $\xi \in \mathcal{D}$,

$$
\left\langle X_{B} \xi \mid \xi\right\rangle_{B}=\langle X \xi \mid \xi\rangle \geq 0, \quad \forall B \succeq A .
$$

Since $\mathcal{D}$ is dense in $\mathcal{H}_{B}$, we have $\left\langle X_{B} \xi \mid \xi\right\rangle_{B} \geq 0, \forall \xi \in \mathcal{H}_{B}$.

(ii) $\Rightarrow\left(\right.$ i): Let $X_{B} \geq 0$ for every $B \succeq A$. Then, for every $\xi \in \mathcal{D},\langle X \xi \mid \xi\rangle=$ $\left\langle X_{B} \xi \mid \xi\right\rangle_{B} \geq 0$.

Theorem 3.5. Let $X \in \mathfrak{L}_{B}\left(\mathcal{D}, \mathcal{D}^{\times}\right)$. The following statements are equivalent.

(i) $X: \mathcal{D} \rightarrow \mathcal{H}$ and $\bar{X} \in \mathcal{B}(\mathcal{H})$.

(ii) $X \in \mathfrak{L}_{B}\left(\mathcal{D}, \mathcal{D}^{\times}\right)_{b}$.

(iii) There exists $\lambda>0$ such that

$$
-\lambda I \leq \Re(X) \leq \lambda I, \quad-\lambda I \leq \Im(X) \leq \lambda I
$$

where $\Re(X)=\frac{X+X^{\dagger}}{2}$ and $\Im(X)=\frac{X-X^{\dagger}}{2 i}$.

Proof. (i) $\Rightarrow$ (ii): If $X: \mathcal{D} \rightarrow \mathcal{H}$ and $X$ is bounded, then, for every $A \in \mathcal{L}^{\dagger}(\mathcal{D})$,

$$
|\langle X \xi \mid \eta\rangle| \leq\|\bar{X}\|\|\xi\|\|\eta\| \leq\|\bar{X}\|\|\xi\|_{A}\|\eta\|_{A} .
$$


This means that $X$ has a bounded representative $X_{A}$ in every $\mathcal{B}\left(\mathcal{H}_{A}\right)$. By (2), $\left\|X_{A}\right\|_{A, A} \leq\|\bar{X}\|$, for every $A \in \mathcal{L}^{\dagger}(\mathcal{D})$, so $\sup _{A \in \mathcal{L}^{\dagger}(\mathcal{D})}\left\|X_{A}\right\|_{A, A}<+\infty$.

(ii) $\Rightarrow$ (i) Let $X \in \mathfrak{L}_{\mathrm{B}}\left(\mathcal{D}, \mathcal{D}^{\times}\right)_{b}$. Then, for every $A \in \mathcal{L}^{\dagger}(\mathcal{D})$

$$
|\langle X \xi \mid \eta\rangle| \leq\left\|X_{A}\right\|_{A, A}\|\xi\|_{A}\|\eta\|_{A}, \quad \forall \xi, \eta \in \mathcal{D} .
$$

In particular, for $A=0$,

$$
|\langle X \xi \mid \eta\rangle| \leq\left\|X_{0}\right\|\|\xi\|\|\eta\|, \quad \forall \xi, \eta \in \mathcal{D} .
$$

By (3), for every $\xi \in \mathcal{D}, F(\eta)=\langle X \xi \mid \eta\rangle$ is a bounded conjugate linear functional on $\mathcal{D}$, so by Riesz's lemma $X \xi \in \mathcal{H}$. It is, finally easily seen that $\bar{X} \in \mathcal{B}(\mathcal{H})$.

(iii) $\Rightarrow$ (i) Suppose first that $X=X^{\dagger}$. Note that the operator $X$ satisfies the following: $0 \leq \frac{X+\lambda I}{2 \lambda} \leq I$; so $\frac{X+\lambda I}{2 \lambda}$ is a positive operator and $\left\langle\frac{X+\lambda I}{2 \lambda} \xi \mid \xi\right\rangle \leq\langle\xi \mid \xi\rangle, \forall \xi \in \mathcal{D}$; this implies that

$$
\left|\left\langle\frac{X+\lambda I}{2 \lambda} \xi \mid \eta\right\rangle\right| \leq\|\xi\|\|\eta\|, \quad \forall \xi, \eta \in \mathcal{D}
$$

and by Riesz's lemma there exists $\zeta \in \mathcal{H}$ such that

$$
\left\langle\frac{X+\lambda I}{2 \lambda} \xi \mid \eta\right\rangle=\langle\zeta \mid \eta\rangle, \quad \forall \xi, \eta \in \mathcal{D}
$$

and then $\frac{X+\lambda I}{2 \lambda} \xi \in \mathcal{H}$. This implies that $X \xi \in \mathcal{H}$ too. Moreover, $X$ has a representative for every $A \in \mathcal{L}^{\dagger}(\mathcal{D})$. Indeed,

$$
|\langle X \xi \mid \eta\rangle| \leq \gamma\|\xi\|\|\eta\| \leq \gamma\|\xi\|_{A}\|\eta\|_{A} \quad \forall A \in \mathcal{L}^{\dagger}(\mathcal{D}),
$$

where $\gamma>0$. From (41) it follows that $X$ is bounded and $\bar{X} \in \mathcal{B}(\mathcal{H})$. In the very same way one can prove the boundedness of $X$ if $X^{\dagger}=-X$. The result for a general $X$ follows easily.

(i) $\Rightarrow$ (iii): this is a standard result of the $\mathrm{C}^{*}$-algebras theory.

\section{Bounded Elements of C*-InduCtive LOCALly CONVEX SPACES}

The results obtained in Section 3 have an abstract generalization to locally convex spaces that are inductive limits of $\mathrm{C}^{*}$-algebras in a generalized sense. These spaces were called $C^{*}$-inductive locally convex spaces in [8]. We begin with recalling the basic definitions.

Let $\mathfrak{A}$ be a vector space over $\mathbb{C}$. Let $\mathbb{F}$ be a set of indices directed upward and consider, for every $\alpha \in \mathbb{F}$, a Banach space $\mathfrak{A}_{\alpha} \subset \mathfrak{A}$ such that:

(I.1) $\mathfrak{A}_{\alpha} \subseteq \mathfrak{A}_{\beta}$, if $\alpha \leq \beta$;

(I.2) $\mathfrak{A}=\bigcup_{\alpha \in \mathbb{F}} \mathfrak{A}_{\alpha}$; 
(I.3) $\forall \alpha \in \mathbb{F}$, there exists a $\mathrm{C}^{*}$-algebra $\mathfrak{B}_{\alpha}$ (with unit $e_{\alpha}$ and norm $\|\cdot\|_{\alpha}$ ) and a norm-preserving isomorphism of vector spaces $\phi_{\alpha}: \mathfrak{B}_{\alpha} \rightarrow \mathfrak{A}_{\alpha}$; (I.4) $x_{\alpha} \in \mathfrak{B}_{\alpha}^{+} \Rightarrow x_{\beta}=\left(\phi_{\beta}^{-1} \phi_{\alpha}\right)\left(x_{\alpha}\right) \in \mathfrak{B}_{\beta}^{+}$, for every $\alpha, \beta \in \mathbb{F}$ with $\beta \geq \alpha$.

We put $j_{\beta \alpha}=\phi_{\beta}^{-1} \phi_{\alpha}$, if $\alpha, \beta \in \mathbb{F}, \beta \geq \alpha$.

If $x \in \mathfrak{A}$, there exist $\alpha \in \mathbb{F}$ such that $x \in \mathfrak{A}_{\alpha}$ and (a unique) $x_{\beta} \in \mathfrak{B}_{\beta}$ such that $x=\phi_{\beta}\left(x_{\beta}\right)$, for all $\beta \geq \alpha$.

Then, we put

$$
j_{\beta \alpha}\left(x_{\alpha}\right):=x_{\beta} \quad \text { if } \alpha \leq \beta .
$$

By (I.4), it follows easily that $j_{\beta \alpha}$ preserves the involution; i.e., $j_{\beta \alpha}\left(x_{\alpha}^{*}\right)=$ $\left(j_{\beta \alpha}\left(x_{\alpha}\right)\right)^{*}$.

The family $\left\{\mathfrak{B}_{\alpha}, j_{\beta \alpha}, \beta \geq \alpha\right\}$ is a directed system of $C^{*}$-algebras, in the sense that:

(J.1) for every $\alpha, \beta \in \mathbb{F}$, with $\beta \geq \alpha, j_{\beta \alpha}: \mathfrak{B}_{\alpha} \rightarrow \mathfrak{B}_{\beta}$ is a linear and injective map; $j_{\alpha \alpha}$ is the identity of $\mathfrak{B}_{\alpha}$,

(J.2) for every $\alpha, \beta \in \mathbb{F}$, with $\alpha \leq \beta, \phi_{\alpha}=\phi_{\beta} j_{\beta \alpha}$.

(J.3) $j_{\gamma \beta} j_{\beta \alpha}=j_{\gamma \alpha}, \alpha \leq \beta \leq \gamma$.

We assume that, in addition, the $j_{\beta \alpha}$ 's are Schwarz maps (see, e.g. [15]); i.e.,

(sch) $j_{\beta \alpha}\left(x_{\alpha}\right)^{*} j_{\beta \alpha}\left(x_{\alpha}\right) \leq j_{\beta \alpha}\left(x_{\alpha}^{*} x_{\alpha}\right), \quad \forall x_{\alpha} \in \mathfrak{B}_{\alpha}, \alpha \leq \beta$.

For every $\alpha, \beta \in \mathbb{F}$, with $\alpha \leq \beta, j_{\beta \alpha}$ is continuous [15] and, moreover,

$$
\left\|j_{\beta \alpha}\left(x_{\alpha}\right)\right\|_{\beta} \leq\left\|x_{\alpha}\right\|_{\alpha}, \quad \forall x_{\alpha} \in \mathfrak{B}_{\alpha} .
$$

An involution in $\mathfrak{A}$ is defined as follows.. Let $x \in \mathfrak{A}$. Then $x \in \mathfrak{A}_{\alpha}$, for some $\alpha \in \mathbb{F}$, i.e., $x=\phi_{\alpha}\left(x_{\alpha}\right)$, for a unique $x_{\alpha} \in \mathfrak{B}_{\alpha}$. Put $x^{*}:=\phi_{\alpha}\left(x_{\alpha}^{*}\right)$. Then if $\beta \geq \alpha$, we have

$$
\phi_{\beta}^{-1}\left(x^{*}\right)=\phi_{\beta}^{-1}\left(\phi_{\alpha}\left(x_{\alpha}^{*}\right)\right)=j_{\beta \alpha}\left(x_{\alpha}^{*}\right)=\left(j_{\beta \alpha}\left(x_{\alpha}\right)\right)^{*}=x_{\beta}^{*} .
$$

It is easily seen that the map $x \mapsto x^{*}$ is an involution in $\mathfrak{A}$. Moreover, by the definition itself, it follows that every map $\phi_{\alpha}$ preserves the involution; i.e., $\phi_{\alpha}\left(x_{\alpha}^{*}\right)=\left(\phi_{\alpha}\left(x_{\alpha}\right)\right)^{*}$, for all $x_{\alpha} \in \mathfrak{B}_{\alpha}, \alpha \in \mathbb{F}$.

Definition 4.1. A locally convex vector space $\mathfrak{A}$, with involution ${ }^{*}$, is called a $C^{*}$-inductive locally convex space if

(i) there exists a family $\left\{\left\{\mathfrak{B}_{\alpha}, \phi_{\alpha}\right\}, \alpha \in \mathbb{F}\right\}$, where $\mathbb{F}$ is a direct set and, for every $\alpha \in \mathbb{F}, \mathfrak{B}_{\alpha}$ is a $\mathrm{C}^{*}$-algebra and $\phi_{\alpha}$ is a linear injective map of $\mathfrak{B}_{\alpha}$ into $\mathfrak{A}$, satisfying the above conditions (I.1) - (I.4) and (sch), with $\mathfrak{A}_{\alpha}=\phi_{\alpha}\left(\mathfrak{B}_{\alpha}\right), \alpha \in \mathbb{F}$; 
(ii) $\mathfrak{A}$ is endowed with the locally convex inductive topology $\tau_{\text {ind }}$ generated by the family $\left\{\left\{\mathfrak{B}_{\alpha}, \phi_{\alpha}\right\}, \alpha \in \mathbb{F}\right\}$.

The family $\left\{\left\{\mathfrak{B}_{\alpha}, \phi_{\alpha}\right\}, \alpha \in \mathbb{F}\right\}$ is called the defining system of $\mathfrak{A}$. We notice that the involution is automatically continuous in $\mathfrak{A}\left[\tau_{\text {ind }}\right]$.

A $\mathrm{C}^{*}$-inductive locally convex space has a natural positive cone.

An element $x \in \mathfrak{A}$ is called positive if there exists $\gamma \in \mathbb{F}$ such that $\phi_{\alpha}^{-1}(x) \in$ $\mathfrak{B}_{\alpha}^{+}, \forall \alpha \geq \gamma$.

We denote by $\mathfrak{A}^{+}$the set of all positive elements of $\mathfrak{A}$.

Then,

(i) Every positive element $x \in \mathfrak{A}$ is hermitian; i.e., $x \in \mathfrak{A}_{h}:=\{y \in \mathfrak{A}$ : $\left.y^{*}=y\right\}$.

(ii) $\mathfrak{A}^{+}$is a non empty convex pointed cone; i.e. $\mathfrak{A}^{+} \cap\left(-\mathfrak{A}^{+}\right)=\{0\}$.

(iii) If $\alpha \in \mathbb{F}$ and $x_{\alpha} \in \mathfrak{B}_{\alpha}^{+}, \phi_{\alpha}\left(x_{\alpha}\right)$ is positive.

Moreover, every hermitian element $x=x^{*}$ is the difference of two positive elements, i.e. there exist $x^{+}, x^{-} \in \mathfrak{A}^{+}$such that $x=x^{+}-x^{-}$.

A linear functional $\omega$ is said to be positive if $\omega(x) \geq 0$ for every $x=$ $\left(x_{\alpha}\right) \in \mathfrak{A}$. As shown in [8, Prop. 3.9, 3.10], $\omega$ is positive if, and only if, $\omega_{\alpha}\left(x_{\alpha}\right):=\omega\left(\phi_{\alpha}\left(x_{\alpha}\right)\right) \geq 0$ for every $\alpha \in \mathbb{F}$. We write, in this case, $\omega=\lim _{\longrightarrow} \omega_{\alpha}$.

\subsection{Bounded elements.}

Definition 4.2. Let $\mathfrak{A}$ be a $\mathrm{C}^{*}$-inductive locally convex space. An element $x=\left(x_{\alpha}\right) \in \mathfrak{A}$, with $x_{\alpha} \in \mathfrak{B}_{\alpha}$, is called bounded if $x \in \mathfrak{A}_{\alpha}$, for every $\alpha \in \mathbb{F}$ and $\sup _{\alpha \in \mathbb{F}}\left\|x_{\alpha}\right\|_{\alpha}<\infty$. The set of bounded elements of $\mathfrak{A}$ is denoted by $\mathfrak{A}_{b}$.

Proposition 4.3. The set $\mathfrak{A}_{b}$ is a Banach space under the norm $\|x\|_{b}=$ $\sup _{\alpha \in \mathbb{F}}\left\|x_{\alpha}\right\|_{\alpha}$.

Proof. We only prove the completeness. Let $\left\{x_{n}\right\}$ be a Cauchy sequence in $\mathfrak{A}_{b}$. Then for every $\alpha \in \mathbb{F}$ the sequence $\left\{x_{n}^{\alpha}\right\}$, with $x_{n}^{\alpha}:=\left(x_{n}\right)_{\alpha}$, is Cauchy in $\mathfrak{B}_{\alpha}$ so it converges to some $x_{\alpha} \in \mathfrak{B}_{\alpha}$. Since the $j_{\beta \alpha}$ 's are continuous, one easily proves that the family $\left\{x_{\alpha}\right\}$ defines an element $x=\left(x_{\alpha}\right)$ of $\mathfrak{A}$. From the Cauchy condition, for every $\epsilon>0$, there exists $n_{\epsilon} \in \mathbb{N}$ such that

$$
\sup _{\alpha \in \mathbb{F}}\left\|x_{n}^{\alpha}-x_{m}^{\alpha}\right\|_{\alpha}<\epsilon
$$


If $m>n_{\epsilon}$,

$$
\left\|x_{\alpha}\right\|_{\alpha} \leq\left\|x_{\alpha}-x_{m}^{\alpha}\right\|_{\alpha}+\left\|x_{m}^{\alpha}\right\|_{\alpha} \leq \epsilon+\left\|x_{m}^{\alpha}\right\|_{\alpha} .
$$

Hence,

$$
\sup _{\alpha \in \mathbb{F}}\left\|x_{\alpha}\right\|_{\alpha} \leq \epsilon+\sup _{\alpha \in \mathbb{F}}\left\|x_{m}^{\alpha}\right\|_{\alpha}<\infty
$$

Thus $x \in \mathfrak{A}_{b}$.

Fix now $n>n_{\epsilon}$ and let $m \rightarrow \infty$ in (6). Then,

$$
\sup _{\alpha \in \mathbb{F}}\left\|x_{n}^{\alpha}-x_{\alpha}\right\|_{\alpha} \leq \epsilon
$$

This proves that $x_{n} \rightarrow x$.

In what follows we will consider *-representations of a $\mathrm{C}^{*}$-inductive locally convex space. We recall the basic definitions.

Let $\mathbb{F}$ be a set directed upward by $\leq$. A family $\left\{\mathcal{H}_{\alpha}, U_{\beta \alpha}, \alpha, \beta \in \mathbb{F}, \beta \geq \alpha\right\}$, where each $\mathcal{H}_{\alpha}$ is a Hilbert space (with inner product $\langle\cdot \mid \cdot\rangle_{\alpha}$ and norm $\|\cdot\|_{\alpha}$ ) and, for every $\alpha, \beta \in \mathbb{F}$, with $\beta \geq \alpha, U_{\beta \alpha}$ is a linear map from $\mathcal{H}_{\alpha}$ into $\mathcal{H}_{\beta}$, is called a directed contractive system of Hilbert spaces if the following conditions are satisfied

(i) $U_{\beta \alpha}$ is injective;

(ii) $\left\|U_{\beta \alpha} \xi_{\alpha}\right\|_{\beta} \leq\left\|\xi_{\alpha}\right\|_{\alpha}, \quad \forall \xi_{\alpha} \in \mathcal{H}_{\alpha}$;

(iii) $U_{\alpha \alpha}=I_{\alpha}$, the identity of $\mathcal{H}_{\alpha}$;

(iv) $U_{\gamma \alpha}=U_{\gamma \beta} U_{\beta \alpha}, \alpha \leq \beta \leq \gamma$.

A directed contractive system of Hilbert spaces defines a conjugate dual pair $\left(\mathcal{D}^{\times}, \mathcal{D}\right)$ which is called the joint topological limit [7] of the directed contractive system $\left\{\mathcal{H}_{\alpha}, U_{\beta \alpha}, \alpha, \beta \in \mathbb{F}, \beta \geq \alpha\right\}$ of Hilbert spaces.

Definition 4.4. Let $\mathfrak{A}$ be the $\mathrm{C}^{*}$-inductive locally convex space defined by the system $\left\{\left\{\mathfrak{B}_{\alpha}, \Phi_{\alpha}\right\}, \alpha \in \mathbb{F}\right\}$ as in Definition 4.1.

For each $\alpha \in \mathbb{F}$, let $\pi_{\alpha}$ be a ${ }^{*}$-representation of $\mathfrak{B}_{\alpha}$ in Hilbert space $\mathcal{H}_{\alpha}$. The collection $\pi:=\left\{\pi_{\alpha}\right\}$ is said to be a ${ }^{*}$-representation of $\mathfrak{A}$ if

(i) for every $\alpha, \beta \in \mathbb{F}$ there exists a linear map $U_{\beta \alpha}: \mathcal{H}_{\alpha} \rightarrow \mathcal{H}_{\beta}$ such that the family $\left\{\mathcal{H}_{\alpha}, U_{\beta \alpha}, \alpha, \beta \in \mathbb{F}, \beta \geq \alpha\right\}$ is a directed contractive system of Hilbert spaces;

(ii) the following equality holds

$$
\pi_{\beta}\left(j_{\beta \alpha}\left(x_{\alpha}\right)\right)=U_{\beta \alpha} \pi_{\alpha}\left(x_{\alpha}\right) U_{\beta \alpha}^{*}, \quad \forall x_{\alpha} \in \mathfrak{B}_{\alpha}, \beta \geq \alpha .
$$


In this case we write $\pi(x)=\lim _{\longrightarrow} \pi_{\alpha}\left(x_{\alpha}\right)$ for every $x=\left(x_{\alpha}\right) \in \mathfrak{A}$ or, for short, $\pi=\underline{\lim } \pi_{\alpha}$.

The *-representation $\pi$ is said to be faithful if $x \in \mathfrak{A}^{+}$and $\pi(x)=0$ imply $x=0$ (of course, $\pi(x)=0$ means that there exists $\gamma \in \mathbb{F}$ such that $\pi_{\alpha}\left(x_{\alpha}\right)=0$, for $\left.\alpha \geq \gamma\right)$.

Remark 4.5. With this definition (which is formally different from that given in [8] but fully equivalent), $\pi(x), x \in \mathfrak{A}$, is not an operator but rather a collection of operators. But as shown in [8], $\pi(x)$ can be regarded as an operator acting on the joint topological limit $\left(\mathcal{D}^{\times}, \mathcal{D}\right)$ of $\left\{\mathcal{H}_{\alpha}, U_{\beta \alpha}, \alpha, \beta \in\right.$ $\mathbb{F}, \beta \geq \alpha\}$. The corresponding space of operators was denoted by $L_{\mathrm{B}}\left(\mathcal{D}, \mathcal{D}^{\times}\right)$; it behaves in the very same way as the space $\mathfrak{L}_{B}\left(\mathcal{D}, \mathcal{D}^{\times}\right)$studied in Section 3 and reduces to it when the family of Hilbert spaces is exactly $\left\{\mathcal{H}_{A} ; A \in\right.$ $\left.\mathcal{L}^{\dagger}(\mathcal{D})\right\}$. The main difference consists in the fact that the $\mathcal{H}_{\alpha}$ 's need not be all subspaces of a certain Hilbert space $\mathcal{H}$.

Lemma 4.6. Let $\pi=\lim _{\alpha} \pi_{\alpha}$ be a faithful ${ }^{*}$-representation of $\mathfrak{A}$. Then, for every $\alpha \in \mathbb{F}, \pi_{\alpha}$ is a faithful ${ }^{*}$-representation of $\mathfrak{B}_{\alpha}$.

Proof. Let $x_{\alpha} \in \mathfrak{B}_{\alpha}^{+}$with $\pi_{\alpha}\left(x_{\alpha}\right)=0$. Let $x \in \mathfrak{A}$ be the unique element of $\mathfrak{A}$ such that $x=\phi_{\alpha}\left(x_{\alpha}\right)$. Then $\pi_{\beta}\left(x_{\beta}\right)=\pi_{\beta}\left(j_{\beta \alpha}\left(x_{\alpha}\right)\right)=U_{\beta \alpha} \pi_{\alpha}\left(x_{\alpha}\right) U_{\beta \alpha}^{*}=0$. Hence $\pi(x)=0$ and, therefore $x=0$. Thus there exists $\bar{\gamma} \in \mathbb{F}$ such that $x_{\gamma}=0$, for $\gamma \geq \bar{\gamma}$. Let $\beta \geq \alpha, \bar{\gamma}$. Then $0=x_{\beta}=j_{\beta \alpha}\left(x_{\alpha}\right)$. Hence, by the injectivity of $j_{\beta \alpha}, x_{\alpha}=0$.

As shown in [8, Proposition 3.16], if a $\mathrm{C}^{*}$-inductive locally convex space $\mathfrak{A}$ fulfills the following conditions

$\left(r_{1}\right)$ if $x_{\alpha} \in \mathfrak{B}_{\alpha}$ and $j_{\beta \alpha}\left(x_{\alpha}\right) \geq 0, \beta \geq \alpha$, then $x_{\alpha} \geq 0$

$\left(r_{2}\right) e_{\beta} \in j_{\beta \alpha}\left(\mathfrak{B}_{\alpha}\right), \quad \forall \alpha, \beta \in \mathbb{F}, \beta \geq \alpha$;

$\left(r_{3}\right)$ every positive linear functional $\omega=\lim _{\longrightarrow} \omega_{\alpha}$ on $\mathfrak{A}$ satisfies the following property

- if $\alpha \in \mathbb{F}$ and $\omega_{\beta}\left(j_{\beta \alpha}\left(x_{\alpha}^{*}\right) j_{\beta \alpha}\left(x_{\alpha}\right)\right)=0$, for some $\beta>\alpha$ and $x_{\alpha} \in \mathfrak{B}_{\alpha}$, then $\omega_{\alpha}\left(x_{\alpha}^{*} x_{\alpha}\right)=0 ;$

then, $\mathfrak{A}$ admits a faithful representation. The conditions $\left(r_{1}\right),\left(r_{2}\right)$, in fact, guarantee that $\mathfrak{A}$ possesses sufficiently many positive linear functionals, in the sense that for every $x \in \mathfrak{A}^{+}, x \neq 0$ there exists a positive linear functional $\omega$ on $\mathfrak{A}$ such that $\omega(x)>0[8$, Theorem 3.14].

Theorem 4.7. Let $\mathfrak{A}$ be a $C^{*}$-inductive locally convex space and $x=\left(x_{\alpha}\right) \in$ $\mathfrak{A}$. The following statements hold. 
(i) If $x \in \mathfrak{A}_{b}$, then, for every ${ }^{*}$-representation $\pi=\lim _{\longrightarrow} \pi_{\alpha}$ of $\mathfrak{A}$, one has

$$
\sup _{\alpha \in \mathbb{F}}\left\|\pi_{\alpha}\left(x_{\alpha}\right)\right\|_{\alpha \alpha}<\infty
$$

where $\|\cdot\|_{\alpha \alpha}$ denote the norm of $\mathfrak{B}\left(\mathcal{H}_{\alpha}\right)$.

(ii) Conversely, if $\mathfrak{A}$ admits a faithful ${ }^{*}$-representation $\pi^{f}=\underline{\lim _{\longrightarrow}} \pi_{\alpha}^{f}$ and

$$
\sup _{\alpha \in \mathbb{F}}\left\|\pi_{\alpha}^{f}\left(x_{\alpha}\right)\right\|_{\alpha \alpha}<\infty
$$

then $x \in \mathfrak{A}_{b}$.

Proof. (i): For every $\alpha \in \mathbb{F}, \pi_{\alpha}$ is a ${ }^{*}$-representation of the $\mathrm{C}^{*}$-algebra $\mathfrak{B}_{\alpha}$. Hence

$$
\left\|\pi_{\alpha}\left(x_{\alpha}\right)\right\|_{\alpha \alpha} \leq\left\|x_{\alpha}\right\|_{\alpha} .
$$

Thus if $x \in \mathfrak{A}_{b}$ the statement follows immediately from the definition.

(ii): Let $\pi^{f}(x)=\lim _{\alpha} \pi_{\alpha}^{f}\left(x_{\alpha}\right)$. Then, by Lemma 4.6, for every $\alpha \in \mathbb{F}, \pi_{\alpha}^{f}$ is a faithful representation of $\mathfrak{B}_{\alpha}$. The ${ }^{*}$-representation $\pi_{\alpha}^{f}$ is an isometric isomorphism of $\mathrm{C}^{*}$-algebras, for all $\alpha \in \mathbb{F}$; hence

$$
\sup _{\alpha \in \mathbb{F}}\left\|x_{\alpha}\right\|_{\alpha}=\sup _{\alpha \in \mathbb{F}}\left\|\pi_{\alpha}^{f}\left(x_{\alpha}\right)\right\|_{\alpha \alpha}<\infty .
$$

This proves that $x$ is a bounded element of $\mathfrak{A}$.

4.2. Order bounded elements. Let $\mathfrak{A}$ be a $\mathrm{C}^{*}$-inductive locally convex space. If $x \in \mathfrak{A}$, we put

$$
\Re(x)=\frac{x+x^{*}}{2} \text { and } \Im(x)=\frac{x-x^{*}}{2 i} .
$$

Both $\Re(x)$ and $\Im(x)$ are symmetric elements of $\mathfrak{A}$.

Assume that $\mathfrak{A}$ has an element $u=u^{*}$ such that $\left\|u_{\alpha}\right\|_{\alpha} \leq 1$, for every $\alpha \in \mathbb{F}$, and there exists $\gamma \in \mathbb{F}$ such that $u_{\beta}=j_{\beta \gamma}\left(e_{\gamma}\right) \forall \beta \geq \gamma,\left(e_{\gamma}\right.$ is the unit of $\mathfrak{B}_{\gamma}$ ). For shortness we call the element $u$ a pre-unit of $\mathfrak{A}$.

Remark 4.8. The pre-unit $u \in \mathfrak{A}$, if any, is unique. Indeed, let suppose there is another $v \in \mathfrak{A}$ satisfying the same properties as $u$. Then,

$$
\exists \gamma, \gamma^{\prime} \in \mathbb{F} ; u_{\beta}=j_{\beta \gamma}\left(e_{\gamma}\right), v_{\beta^{\prime}}=j_{\beta^{\prime} \gamma^{\prime}}\left(e_{\gamma}^{\prime}\right), \quad \forall \beta \geq \gamma, \beta^{\prime} \geq \gamma^{\prime}
$$

so, if $\delta \geq \gamma, \gamma^{\prime}$, one has $u_{\lambda}=v_{\lambda}, \forall \lambda \geq \delta$.

Definition 4.9. Let $\mathfrak{A}$ be a $\mathrm{C}^{*}$-inductive locally convex space with pre-unit $u$. We say that $x \in \mathfrak{A}$ is order bounded (with respect to $u$ ) if there exists $\lambda>0$ such that

$$
-\lambda u \leq \Re(x) \leq \lambda u \quad-\lambda u \leq \Im(x) \leq \lambda u .
$$


Theorem 4.10. Let $\mathfrak{A}$ be a $C^{*}$-inductive locally convex space satisfying condition $\left(\mathrm{r}_{1}\right)$. Assume that $\mathfrak{A}$ has a pre-unit $u$.

Then, $x \in \mathfrak{A}_{b}$ if, and only if, $x$ has a representative for every $\alpha \in \mathbb{F}$ (i.e. for every $\alpha \in \mathbb{F}$, there exists $x_{\alpha} \in \mathfrak{B}_{\alpha}$ such that $x=\phi_{\alpha}\left(x_{\alpha}\right)$ ) and $x$ is order bounded with respect $u$.

Proof. Let us assume that $x=x^{*} \in \mathfrak{A}_{b}$. Then, $x$ has a representative $x_{\alpha}$, with $x_{\alpha}^{*}=x_{\alpha}$, in every $\mathfrak{B}\left(\mathcal{H}_{\alpha}\right)$ and $\lambda:=\sup _{\alpha \in \mathbb{F}}\left\|x_{\alpha}\right\|_{\alpha}<\infty$. Hence, we have

$$
-\lambda e_{\alpha} \leq x_{\alpha} \leq \lambda e_{\alpha}, \quad \forall \alpha \in \mathbb{F},
$$

where $e_{\alpha}$ denotes the unit of $\mathfrak{B}_{\alpha}$. By the definition of $u$, there exists $\gamma \in \mathbb{F}$ such that $u_{\beta}=j_{\beta \gamma}\left(e_{\gamma}\right)$ for $\beta \geq \gamma$. Hence, taking into account that the maps $j_{\beta \alpha}$ preserve the order, we have

$$
-\lambda u_{\beta} \leq x_{\beta} \leq \lambda u_{\beta}, \quad \forall \beta \geq \gamma .
$$

This implies that $-\lambda u \leq x \leq \lambda u$.

Now, let us suppose that for some $\lambda>0,-\lambda u \leq x \leq \lambda u$. Then, there exists $\gamma \in \mathbb{F}$ such that

$$
-\lambda u_{\beta} \leq x_{\beta} \leq \lambda u_{\beta}, \quad \forall \beta \geq \gamma .
$$

Let now $\alpha \in \mathbb{F}$. Then, there is $\delta \geq \alpha, \gamma$ such that (8) holds for $\delta \geq \alpha$. Hence, by using $\left(r_{1}\right)$, we conclude that

$$
-\lambda u_{\alpha} \leq x_{\alpha} \leq \lambda u_{\alpha} \quad \forall \alpha \in \mathbb{F} .
$$

This implies that, $\left\|x_{\alpha}\right\|_{\alpha} \leq \lambda$, for every $\alpha \in \mathbb{F}$. Thus, $x \in \mathfrak{A}_{b}$.

From the proof of the previous theorem it follows easily that

Proposition 4.11. Let $x=x^{*} \in \mathfrak{A}_{b}$ and put

$$
p(x)=\inf \{\lambda>0 ;-\lambda u \leq x \leq \lambda u\} .
$$

Then, $p(x)=\|x\|_{b}$.

\section{C*-INDUCTIVE PARTial *-ALgEBRas}

As shown in [8], a partial multiplication in $\mathfrak{A}$ can be defined by a family $w=\left\{w_{\alpha}\right\}, w_{\alpha} \in \mathfrak{B}_{\alpha}$. Let $w=\left\{w_{\alpha}\right\}$ be a family of elements, such that each $w_{\alpha} \in \mathfrak{B}_{\alpha}^{+}$and $j_{\beta \alpha}\left(w_{\alpha}\right)=w_{\beta}$, for all $\alpha, \beta \in \mathbb{F}$ with $\beta \geq \alpha$.

Let $x, y \in \mathfrak{A}$. The partial multiplication $x \cdot y$ is defined by the conditions:

$$
\begin{aligned}
& \exists \gamma \in \mathbb{F}: \phi_{\beta}\left(\phi_{\beta}^{-1}(x) w_{\beta} \phi_{\beta}^{-1}(y)\right)=\phi_{\beta^{\prime}}\left(\phi_{\beta^{\prime}}^{-1}(x) w_{\beta^{\prime}} \phi_{\beta^{\prime}}^{-1}(y)\right), \forall \beta, \beta^{\prime} \geq \gamma \\
& x \cdot y=\phi_{\beta}\left(\phi_{\beta}^{-1}(x) w_{\beta} \phi_{\beta}^{-1}(y)\right), \quad \beta \geq \gamma .
\end{aligned}
$$


Then, $\mathfrak{A}$ is an associative partial *-algebra with respect to the usual operations and the above defined multiplication (see [2, Sect. 2.1.1] for the definitions) and we will call it a $C^{*}$-inductive partial *-algebra.

The partial *-algebra $\mathfrak{A}$ has a unit $e$ (that is, an element $e$ which is a leftand right universal multiplier such that $x \cdot e=e \cdot x=x$, for every $x \in \mathfrak{A}$ ) if, and only if, every element $w_{\alpha}$ of the family $\left\{w_{\alpha}\right\}$ defining the multiplication is invertible and

$$
j_{\beta \alpha}\left(w_{\alpha}^{-1}\right)=w_{\beta}^{-1}, \quad \forall \alpha, \beta \in \mathbb{F}, \beta \geq \alpha .
$$

In this case, $e=\phi_{\alpha}\left(w_{\alpha}^{-1}\right)$, independently of $\alpha \in \mathbb{F}$.

The element $e$ is called a bounded unit if it is a bounded element of $\mathfrak{A}$ and $\|e\|_{b}=1$.

Proposition 5.1. Let $\mathfrak{A}$ be a $C^{*}$-inductive partial *-algebra with the multiplication defined by a family $\left\{w_{\alpha}\right\}$. Assume that $e=\left(w_{\alpha}^{-1}\right)$ is a bounded unit of $\mathfrak{A}$. Then $\mathfrak{A}_{b}$ is a Banach partial *-algebra; that is, $\mathfrak{A}_{b}\left[\|\cdot\|_{b}\right]$ is a Banach space with isometric involution * and there exists $C \geq 1$ such that the following inequality holds

$$
\|x \cdot y\|_{b} \leq C\|x\|_{b}\|y\|_{b}, \quad \forall x, y \in \mathfrak{A}_{b} \text { with } x \cdot y \text { well-defined. }
$$

Remark 5.2. The constant $C$ in (10) can be taken equal to 1 if $w_{\alpha}^{-1}=e_{\alpha}$, for each $\alpha \in \mathbb{F}$, where $e_{\alpha}$ is the unit of the $\mathrm{C}^{*}$-algebra $\mathfrak{B}_{\alpha}$. Under the same assumption, the norm of $\mathfrak{A}_{b}$ satisfies the $\mathrm{C}^{*}$-property, which in our case reads

$$
\left\|x^{*} \cdot x\right\|_{b}=\|x\|_{b}^{2}, \quad \forall x \in \mathfrak{A}_{b} \text { with } x^{*} \cdot x \text { well-defined. }
$$

This is no longer true in the general case.

Remark 5.3. In Example 5.3 of 8 ] two of us tried to construct a family $\left\{W_{A} \in \mathfrak{B}\left(\mathcal{H}_{A}\right) ; A \in \mathcal{L}^{\dagger}(\mathcal{D})\right\}$ so that the partial multiplication defined in $\mathfrak{L}_{\mathrm{B}}\left(\mathcal{D}, \mathcal{D}^{\times}\right)$by the method mentioned above would reproduce the quasi *algebra structure of $\left(\mathfrak{L}_{\mathrm{B}}\left(\mathcal{D}, \mathcal{D}^{\times}\right), \mathcal{L}^{\dagger}(\mathcal{D})\right)$ (see Section 2). Unfortunately, the conclusion of that discussion is uncorrect (see, 9] for more details).

Let $\mathfrak{A}$ be a $\mathrm{C}^{*}$-inductive partial *-algebra with the multiplication defined by a family $\left\{w_{\alpha}\right\}$ as above. The spaces $R \mathfrak{A}$ and $L \mathfrak{A}$ of the right-, respectively, left universal multipliers (with respect to $w$ ) of $\mathfrak{A}$ are algebras. Hence, $\mathfrak{A}_{0}:=L \mathfrak{A} \cap R \mathfrak{A}$ is a ${ }^{*}$-algebra and, thus,

(i) $\left(\mathfrak{A}, \mathfrak{A}_{0}\right)$ is a quasi *-algebra.

(ii) If $\mathfrak{A}$ is endowed with $\tau_{\text {ind }}$, then the maps $x \mapsto x^{*}, x \mapsto a \cdot x, x \mapsto x \cdot b$, $a, b \in \mathfrak{A}_{0}$ are continuous. 
It is easily seen from the very definition that, if $a \in R \mathfrak{A}$ and $x \in \mathfrak{A}^{+}$, then $a^{*} x a \in \mathfrak{A}^{+}$. Hence, if $\mathcal{P}(\mathfrak{A})$ denotes the family of all positive linear functionals on $\mathfrak{A}$, we have in particular $\omega\left(a^{*} x a\right) \geq 0$, for every $\omega \in \mathcal{P}(\mathfrak{A})$.

Theorem 5.4. Let $\mathfrak{A}$ be a $C^{*}$-inductive partial ${ }^{*}$-algebra with the multiplication defined by a family $\left\{w_{\alpha}\right\}$ and with pre-unit $u$. Assume, moreover, that the following condition (P) holds:

(P) $y \in \mathfrak{A}, \omega\left(a^{*} y a\right) \geq 0, \forall \omega \in \mathcal{P}(\mathfrak{A})$ and $a \in R \mathfrak{A} \Rightarrow y \in \mathfrak{A}^{+}$;

then, for $x \in \mathfrak{A}$, the following conditions are equivalent.

(i) $x$ is order bounded with respect to $u$.

(ii) There exists $\gamma_{x}>0$ such that

$$
\left|\omega\left(a^{*} x a\right)\right| \leq \gamma_{x} \omega\left(a^{*} u a\right), \quad \forall \omega \in \mathcal{P}(\mathfrak{A}), \quad \forall a \in R \mathfrak{A} .
$$

(iii) There exists $\gamma_{x}>0$ such that

$$
\left|\omega\left(b^{*} x a\right)\right|^{2} \leq \gamma_{x} \omega\left(a^{*} u a\right) \omega\left(b^{*} u b\right), \quad \forall \omega \in \mathcal{P}(\mathfrak{A}), \forall a, b \in R \mathfrak{A} .
$$

Proof. It is sufficient to consider the case $x=x^{*}$;

(i) $\Rightarrow$ (ii): Let $\omega \in \mathcal{P}(\mathfrak{A})$. By the hypothesis, $-\gamma u \leq x \leq \gamma u$, for some $\gamma>0$; then $\omega(\gamma u-x) \geq 0$ and $\omega\left(a^{*}(\gamma u-x) a\right) \geq 0, \forall a \in R \mathfrak{A}$. On the other hand, similarly, one can show that $\omega\left(a^{*}(x-\gamma u) a\right) \geq 0$.

(ii) $\Rightarrow(\mathrm{i})$ : Assume now that $u$ is a pre-unit and there exists $\gamma_{x}>0$ such that

$$
\left|\omega\left(a^{*} x a\right)\right| \leq \gamma_{x} \omega\left(a^{*} u a\right), \quad \forall \omega \in \mathcal{P}(\mathfrak{A}), \quad a \in R \mathfrak{A} .
$$

Then

$$
\gamma_{x} \omega\left(a^{*} u a\right) \pm \omega\left(a^{*} x a\right) \geq 0 \Rightarrow \omega\left(a^{*}\left(\gamma_{x} u \pm x\right) a\right) \geq 0, \quad \forall \omega \in \mathcal{P}(\mathfrak{A}), a \in R \mathfrak{A} .
$$

So, by $(\mathrm{P}), \gamma_{x} u \pm x \geq 0$.

(i) $\Rightarrow$ (iii): By the assumption, there exists $\gamma>0$ such that $-\gamma u \leq x \leq \gamma u$. Let $\omega \in \mathcal{P}(\mathfrak{A})$. Then, the linear functional $\omega_{a}$ on $\mathfrak{A}$, defined by $\omega_{a}(x):=$ $\omega\left(a^{*} x a\right)$, is positive. Hence, if $x=x^{*}$

$$
-\gamma \omega_{a}(u) \leq \omega_{a}(x) \leq \gamma \omega_{a}(u)
$$

i.e.,

$$
\left|\omega\left(a^{*} x a\right)\right| \leq \gamma \omega\left(a^{*} u a\right)
$$

Now, let $x \in \mathfrak{A}^{+}, a, b \in R \mathfrak{A}$. Let us define $\Omega_{\omega}^{x}(a, b):=\omega\left(b^{*} x a\right)$. Then, it is easily checked that $\Omega_{\omega}^{x}$ is a positive sesquilinear form on $R \mathfrak{A} \times R \mathfrak{A}$. Using 
the the Cauchy-Schwartz inequality we obtain

$$
\begin{aligned}
\left|\omega\left(b^{*} x a\right)\right| & \leq \omega\left(a^{*} x a\right)^{1 / 2} \omega\left(b^{*} x b\right)^{1 / 2} \\
& \leq \gamma \omega\left(a^{*} u a\right)^{1 / 2} \omega\left(b^{*} u b\right)^{1 / 2} .
\end{aligned}
$$

The extension to arbitrary $x \in \mathfrak{A}$ goes through as in the proof of Proposition 4.3 of $[8]$.

(iii) $\Rightarrow$ (ii) It is trivial.

The previous proof shows that if $x=x^{*} \in \mathfrak{A}$ is order bounded with respect to $u$ then

$$
p(x) \leq \sup \left\{\left|\omega\left(b^{*} x a\right)\right| ; \omega \in \mathcal{P}(\mathfrak{A}) ; a, b \in R \mathfrak{A} ; \omega\left(a^{*} u a\right)=\omega\left(b^{*} u b\right)=1\right\} .
$$

where $p(x)$ is the quantity defined in Proposition 4.11.

The following statement is an easy consequence of Theorem 4.11 and Theorem 5.4 .

Theorem 5.5. Let $\mathfrak{A}$ be a $C^{*}$-inductive partial *-algebra with the multiplication defined by a family $\left\{w_{\alpha}\right\}$ and pre-unit $u$. Assume that conditions $\left(r_{1}\right)$ and $(\mathrm{P})$ are satisfied. For an element $x \in \mathfrak{A}$, having a representative in every $\mathfrak{B}_{\alpha}, \alpha \in \mathbb{F}$, the following statements are equivalent.

(i) $x \in \mathfrak{A}_{b}$.

(ii) $x$ is order bounded with respect to $u$.

(iii) For every $\omega \in \mathcal{P}(\mathfrak{A})$

$$
\left|\omega\left(b^{*} x a\right)\right|^{2} \leq \gamma_{x} \omega\left(a^{*} u a\right) \omega\left(b^{*} u b\right), \quad \forall a, b \in R \mathfrak{A}
$$

\section{REFERENCES}

[1] G.R. Allan, A spectral theory for locally convex algebras, Proc. London. Math. Soc. 15 (1965) 399-421.

[2] J-P. Antoine, A. Inoue, C. Trapani, Partial *-algebras and their operator realizations, Kluwer, Dordrecht, 2002.

[3] J-P. Antoine, C. Trapani, Partial Inner Product Spaces - Theory and Applications, Springer Lecture Notes in Mathematics, vol. 1986, Berlin, Heidelberg, 2009.

[4] J-P. Antoine, C. Trapani, F. Tschinke, Spectral properties of partial *-algebras Mediterr. j. math. 7 (2010) 123-142.

[5] J.-P. Antoine, C.Trapani and F. Tschinke, Bounded elements in certain topological partial *algebras, Studia Math. 203 (2011), 223-251.

[6] J-P. Antoine, G. Bellomonte, C. Trapani, Fully representable and *-semisimple topological partial *-algebras, Studia Mathematica, 208 (2012), 167-194. 
[7] G. Bellomonte, C. Trapani, Rigged Hilbert spaces and contractive families of Hilbert spaces Monatshefte f. Math., 164, (2011) 271-285 (published on line on October 2010 DOI 10.1007/s00605-010-0249-1).

[8] G. Bellomonte, C. Trapani, Quasi *-algebras and generalized inductive limits of $C^{*}$ algebras, Studia Mathematica 202 (2011), 165-190.

[9] G. Bellomonte, C. Trapani, Erratum/Addendum to the paper "Quasi *-algebras and generalized inductive limits of $C^{*}$-algebras", [Studia Mathematica 202 (2011), 165190], to appear.

[10] G. Bellomonte, S. Di Bella, C. Trapani,Operators in Rigged Hilbert spaces: some spectral properties, preprint, Palermo 2013.

[11] M. Fragoulopoulou, C. Trapani and S. Triolo, Locally convex quasi *-algebras with sufficiently many *-representations, J. Math. Anal. Appl. 388 (2012), 1180-1193.

[12] K-D. Kürsten, The completion of the maximal $O_{p}{ }^{*}$-algebra on a Fréchet domain, Publ. Res. Inst. Math. Sci., Kyoto Univ. 22 (1986), 151-175.

[13] K-D. Kürsten, On algebraic properties of partial algebras, Rend. Circ. Mat. Palermo, Ser.II, Suppl. 56 (1998), 111-122.

[14] K-D. Kürsten and M. Läuter, An extreme example concerning factorization products on the Schwartz space $\mathfrak{S}\left(\mathbb{R}^{n}\right)$ Note Mat. 25 (2005/06), 31-38.

[15] T.W. Palmer, Banach Algebras and the General Theory of *-Algebras, Volume 2, Cambridge Univ. Press, Cambridge, 2001.

[16] K. Schmüdgen, Unbounded operator algebras and representation theory, Birkhäuser Verlag, Basel, 1990.

[17] K. Schmüdgen, A strict Positivstellensatz for theWeyl algebra, Math. Ann. 331 (2005) 779-794.

[18] C. Trapani, Bounded elements and spectrum in Banach *-algebras, Studia Mathematica 172 (2006) 249-273.

[19] C.Trapani, Unbounded $C^{*}$-seminorms, biweights and ${ }^{*}$-representations of partial *_ algebras: a review, International J. Math. Math.Sci., Volume 2006 (2006), Article ID 79268, 34 pages.

[20] C. Trapani, Bounded and strongly bounded elements of Banach *-algebras, Contemporary Math. 427 (2007) 417-424.

[21] C. Trapani, *-Representations, seminorms and structure properties of normed quasi *-algebras, Studia Mathematica, 186 (2008), 47-75.

[22] C. Trapani and F. Tschinke, Partial multiplication of operators in rigged Hilbert spaces, Integral Equations Operator Theory 51 (2005), 583-600.

[23] I. Vidav, On some *regular rings, Acad. Serbe Sci. Publ. Inst. Math. 13 (1959) 73-80.

Dipartimento di Matematica e Informatica, Università di Palermo, I-90123

\section{PAlermo, Italy}

E-mail address: bellomonte@math.unipa.it

E-mail address: salvatore.dibella@math.unipa.it

E-mail address: camillo.trapani@unipa.it 